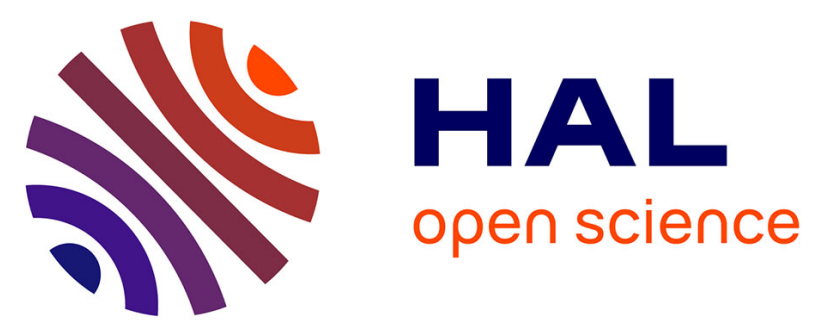

\title{
Fetal Heart Rate Feature Extraction from Cardiotocographic Recordings through Autoregressive Model's Power Spectral-and Pole-based Analysis
}

Alfredo Illanes, Michel Haritopoulos

\section{- To cite this version:}

Alfredo Illanes, Michel Haritopoulos. Fetal Heart Rate Feature Extraction from Cardiotocographic Recordings through Autoregressive Model's Power Spectral-and Pole-based Analysis. 37th annual international conference of the IEEE Engineering in Medicine and Biology Society - EMBC 2015, Aug 2015, Milan, Italy. hal-01203022

\section{HAL Id: hal-01203022 \\ https://hal.science/hal-01203022}

Submitted on 22 Sep 2015

HAL is a multi-disciplinary open access archive for the deposit and dissemination of scientific research documents, whether they are published or not. The documents may come from teaching and research institutions in France or abroad, or from public or private research centers.
L'archive ouverte pluridisciplinaire HAL, est destinée au dépôt et à la diffusion de documents scientifiques de niveau recherche, publiés ou non, émanant des établissements d'enseignement et de recherche français ou étrangers, des laboratoires publics ou privés. 


\title{
Fetal Heart Rate Feature Extraction from Cardiotocographic Recordings through Autoregressive Model's Power Spectral- and Pole-based Analysis
}

\author{
Alfredo Illanes ${ }^{1}$ and Michel Haritopoulos ${ }^{2}$
}

\begin{abstract}
The main objective of this work is to perform an autoregressive model (AR)-based power spectral analysis of the fetal heart rate (FHR) signal for the extraction of significant features for fetal welfare assessment. A group of features is directly computed from the AR-based spectrum while another group is computed from the poles representation. The presented method is applied to real cardiotocographic (CTG) signals and for different frequency bands, and the obtained results are very promising as they exhibit direct correlations between the extracted features and the fetal welfare in terms of umbilical pH.
\end{abstract}

\section{INTRODUCTION}

In current clinical practice, intrapartum monitoring for fetal welfare during labor and delivery is commonly performed by a technology known as electronic fetal monitoring or a cardiotocograph (CTG), which provides continuous information on fetal heart rate (FHR) and maternal uterine contractions (UC). The CTG is a simple and non-invasive tool which can provide to clinicians accurate indicators on fetal status. However, the analysis of the CTG involves interpretation of the complex relationship between the FHR and the UC signals. A good interpretation of CTG depends on the knowledge, skills and experience of healthcare user to interpret FHR and UC signals in order to recognize the different features and patterns of these signals. This leads to significant intra- and inter- observer variations even if specific guidelines have been published for its interpretation [1]. Therefore many efforts have been focused on developing automated techniques to reliably interpret the CTG signal and provide a support system for more objective physicians decisions concerning fetal condition [2], [3]. These approaches involve generally a signal pre-processing step, followed by the extraction of different signal features that constitute the input of a final classification step in order to help distinguish healthy from pathological states. The performance of this last step is completely dependent on the quality of the extracted features.

Several algorithms for CTG feature extraction have been proposed in the literature. Most of them use time domain and frequency domain features related with FHR variability. The

\footnotetext{
*This work was not supported by any organization

${ }^{1}$ Alfredo Illanes is with the Facultad de Ciencias de la Ingeniería, Universidad Austral de Chile, Campus Miraflores General Lagos 2086, Valdivia, Chile alfredoillanes@uach.cl

${ }^{2}$ Michel Haritopoulos is with the PRISME laboratory, EA 4229, Univ. Orléans, ENSI de Bourges, 21 rue de Loigny la Bataille, 28000, Chartres, France Michel.Haritopouloseuniv-orleans.fr
}

time domain features usually consist in statistical indicators computed from short and long term FHR signal analysis [4]. The frequency-based features are usually computed from operations performed over the energy of different spectral components computed with FFT-based techniques [5], [6]. Spectral analysis allows estimating the effect of the interaction between sympathetic and parasympathetic systems on the alterations of the heart rate variation. However, spectral estimation through FFT-based techniques is a tedious task due to the low signal-to-noise ratio (SNR) and to the missing data characterizing CTG recordings. Is for this reason that AR-based parametric modelling techniques have been proposed, because they are known to require only a fraction of the samples required by the FFT method for the same resolution and because they allow the extraction of quantitative spectral parameters. In this context some works propose time-variant AR-based spectral estimation for CTG feature extraction [7]-[9].

The main drawback of these works is that they do not exploit the pole-based representation that AR spectral estimation provides. Moreover, in the case of [7] and [8], the AR spectral estimation is used to investigate the reactivity level of the fetus and not directly focused on separating pathological fetus cases.

The main objective of this work is to extract several AR-based power spectral features from the FHR signal of a CTG recording, and to correlate these features with the fetal welfare in terms of umbilical $\mathrm{pH}$. Hence, two groups of features are analyzed: a first group which is extracted directly from the computed AR-base spectrum and a second one which is made of features extracted from the AR poles model. For experimentation purposes we applied the proposed method to the CTU-UHB CTG Database [10], available on the PhysioNet website, and the obtained results show that several extracted features exhibit a high correlation with the umbilical cord artery $\mathrm{pH}$, which is promising result for further classification steps.

\section{DATA AND METHOD}

\section{A. Experimental Data}

For evaluation of the extracted AR-based power spectral features, the CTU-UHB Intrapartum Cardiotocography Database [10] is used. It is the first open-access database for research purposes on intrapartum CTG signal processing. It contains 552 carefully selected intrapartum recordings providing time information as well as FHR and UC signals, 
both sampled at $4 \mathrm{~Hz}$, and it aims at stimulating research in CTG signal processing and analysis while providing a way to objectively compare results obtained by different approaches. To date, expert obstetrician evaluation of these CTG data is still pending. The aforementioned database is freely available at http://http://physionet.org/physiobank/ database/ctu-uhb-ctgdb/ and the method we propose in this section is applied to these real CTG data. For evaluating the extracted features, the arterial umbilical $\mathrm{pH}$ is used as a gold standard [3].

\section{B. Spectral and Pole Analysis of AR Model for Feature Extraction}

Parametric modeling is a technique for time series analysis in which a mathematical model is fitted to a sampled signal. It has several advantages since it allows description of signals by a few model parameters. The AR model is the preferred method for this class since it is the best compromise between temporal resolution and speed, efficiency and simplicity of algorithms.

An AR model assumes that the value of the current sample $y[n]$ at sample number $n$ in a data sequence, $y[1], y[2], \ldots, y[N]$ can be modeled as a linearly weighted sum of the $p$ most recent sample values, $y[n-1], y[n-$ $2], \ldots, y[n-p]$ and a white zero mean noise $e[n]$ of variance $\sigma^{2}$ :

$$
y[n]=-\sum_{k=1}^{p} a_{k} y[n-k]+e[n]
$$

where $p$ is the model's order and which value is generally chosen to be much smaller than the sequence length $N$, $a_{k}\{k=1,2, \ldots, p\}$ are the $\mathrm{AR}$ parameters and $n$ is the discrete-time index. The z-transform can be applied to (1) and then the AR model transfer function can be expressed as:

$$
H[z]=\frac{Y[z]}{E[z]}=\frac{1}{1+\sum_{k=1}^{p} a_{k} z^{-k}}
$$

where $Y(z)$ and $E(z)$ are the $\mathrm{z}$ transforms of the time series $y[n]$ and the noise $e[n]$, respectively.

The transfer function can be used for several signal analysis, such as Power Spectral Density (PSD) and pole decomposition analyses. The PSD can be computed by evaluating $H(z)$ around the unit circle in the complex plane, i.e., $z=e^{j 2 \pi f}$ :

$$
P S D(f)=\frac{1}{\left|1+\sum_{k=1}^{p} a_{k} e^{-j 2 \pi f k}\right|^{2}} .
$$

The pole representation offers an easier way to comprehend the phenomena and contains important information on the system condition and dynamics, due to the relationship between poles and spectral peaks. The poles $z_{k}$ are obtained by finding the roots of the AR coefficient in the denominator of $H(z)$, giving rise to a pole representation form of (2):

$$
H(z)=\frac{1}{\prod_{k=1}^{p}\left(1-z_{k} z^{-1}\right)} .
$$

Each pair of complex conjugate poles in (4) has a one-toone relationship with a peak in the AR spectrum, $P S D(f)$, in the $\mathrm{z}$ domain [11]. The PSD is divided in bell-shaped curves, with the power and frequency characteristics obtained from the positions and residuals of each pole. The values of each of the $r$ resonant frequencies are given by the phase angle, $\theta_{k}$, of the corresponding pole in the upper half of the complex plane:

$$
f_{k}=\frac{\theta_{k}}{2 \pi}=\tan ^{-1}\left(\frac{\operatorname{Im}\left(z_{k}\right)}{\operatorname{Re}\left(z_{k}\right)}\right) \frac{f_{s}}{2 \pi}
$$

where $f_{s}$ and $f_{k}, k=1,2, \ldots, r$ correspond to the sampling frequency and to the $r$ resonant frequencies resulting from the poles, respectively.

Each pole of frequency $f_{k}$ can be also characterised by its module $m_{k}$ (i.e. how close or far from the unitary circle it is located):

$$
m_{k}=\sqrt{\operatorname{Re}\left(z_{k}\right)^{2}+\operatorname{Im}\left(z_{k}\right)^{2}}
$$

and by the spectral power $P_{k}$, which is obtained from the real part of the residue term $r_{k}$ :

$$
\begin{aligned}
r_{k} & =\left.z^{-1}\left(z-p_{k}\right) H(z)\right|_{z=z_{k}} \\
P_{k} & =\left.2 \sigma^{2} \operatorname{Re}\left(r_{k}\right)\right|_{z=z_{k}} .
\end{aligned}
$$

For the presented work we have experimented various AR model orders (from 8 to 12), but they all provided similar results; finally, we chose a $10^{\text {th }}$ order AR model following the works of [7], [8] and the AR parameters were estimated using the Yule-Walker method.

\section{AR Model Implementation}

1) CTG signal preprocessing: Clinical CTG recordings usually involve several types of artifacts mainly due to mother's and fetus' movements or displacements of the transducer. The loss of sensor's contact can temporarily interrupt one or both the uterine contraction (UC) and the fetal heart rate (FHR) signals. These artifacts result on sharp short duration abrupt changes in the signal or even on completely signal discontinuities thereby causing loss of signal. The main objective of this step is to segment each recorded signal into a number of reliable continuous tracks.

For noise reduction, the artifact rejection method proposed in [3] was applied. In a first step, the segments of the FHR signal that are considered abnormal in amplitude (less than $50 \mathrm{bpm}$ and more than $200 \mathrm{bpm}$ ) or those corresponding to missing data are interpolated using an Hermite spline interpolation. The data are only interpolated when the length of the segment is equal or less than 15 seconds; otherwise, it is removed from the whole signal.

2) Stationary segments extraction: Due to the FHR signal quality and to discontinuities it is always proposed in the literature to extract stationary or stable segments (called also epochs) from the whole FHR signal. The main principle is that spectral estimation increases with data length. However, the state of the fetus change with time. Thus, there is a trade-off between selecting an epoch length that is long 
enough for spectral estimation but also short enough to avoid nonstationarities [9].

In this work a long term analysis is performed following [3], [9]. Segments of 15 minutes are chosen as close as possible to delivery, because during the last minutes major changes in fetal condition can occur [3]. On average, the chosen segments start at approximately $66 \%$ of the beginning of the whole CTG recording.

3) FHR spectrum contributions: Based on previous studies, as for adults, different frequency contributions can be identified in FHR [6], [7]: a DC component (i.e. the average of the FHR), a very low frequency (VLF) band ( $0 \mathrm{~Hz}-0.03 \mathrm{~Hz}$ ), related to very slow control mechanisms and presenting non linear characteristics, low frequency (LF) band $(0.03 \mathrm{~Hz}-0.15 \mathrm{~Hz})$, mainly associated physiologically with neural sympathetic fetal activity, a high frequency (HF) component $(0.5 \mathrm{~Hz}-1.0 \mathrm{~Hz})$ related to fetal breathing $(\mathrm{HF})$, and also a movement frequency $(\mathrm{MF})(0.15 \mathrm{~Hz}-0.5 \mathrm{~Hz})$ correlated with fetal movements and maternal breathing. Their PSDs have been used in previous research work for discriminating fetal pathologies [7].

According to the FHR bands described above, in this work a multi-band analysis and feature extraction algorithm is performed. Each band is extracted from the main FHR signal by using different scales computed with the Daubechies Discrete Wavelet Transform (DWT). The FHR signal is first divided in 10 wavelet scales and then 4 signals are reconstructed whose pseudo-frequency bands are equivalent to:

- Band 1: Total band between $0.01 \mathrm{~Hz}$ and $2 \mathrm{~Hz}$.

- Band 2: Low frequency band between $0.02 \mathrm{~Hz}$ and $0.14 \mathrm{~Hz}$.

- Band 3: Middle frequency band between $0.1 \mathrm{~Hz}$ and $0.4 \mathrm{~Hz}$.

- Band 4: High frequency band between $0.4 \mathrm{~Hz}$ and $1.4 \mathrm{~Hz}$.

4) Extracted indicators: From the FHR AR modeling 20 indicators are extracted. These indicators can be divided in two groups: AR PSD-based and pole-based. The AR PSD-based ones correspond to the more usual indicators proposed for heart rate variability (HRV) analysis, generally FFT-based, as the ones used in [6] and [8] computed from frequency bands' energies, while the pole-based ones are indicators computed directly from the obtained module, residue or frequency of the poles.

a) AR PSD-based indicators: To construct these indicators first the PSD of the AR model is computed and then operations over the energy of the PSD are performed. These indicators were only applied to the high-pass filtered signal (Band 1), because spectral energies require to be computed from the whole frequency range (Band 1). The first five indicators $i n d_{1}, \ldots, i n d_{5}$ are all obtained by computing the definite integrals of the AR model's PSD over $[L B, U B]$ (whose values are summarized in Table I):

$$
\text { ind }_{i}=\frac{1}{T} \int_{L B}^{U B} P S D(f) d f, \quad i \in[1,5],
$$

TABLE I

INTEGRAL LIMITS VALUES FOR INDICATORS 1 TO 5

\begin{tabular}{|c||c|c|c|c|c|}
\hline$i$ & 1 & 2 & 3 & 4 & 5 \\
\hline \hline$L B$ & 0.2 & 1 & 0.15 & 1 & 0.5 \\
\hline UB & 0.03 & 0.2 & 0.04 & 0.5 & 0.15 \\
\hline
\end{tabular}

where for each indicator $i n d_{i}$ the $i$ value is function of the values of the lower $(L B)$ and upper $(U B)$ limits of the integral in (8).

Two indicators $\left(i n d_{\{1,2\}}\right)$ are inspired from work in [8] and the rest of them $\left(i n d_{\{3,4,5\}}\right)$ are similar to the ones used in [6] but with different frequency band limits and computed from the LF, HF and MF bands as described at the beginning of Section II-C.3.

We also used the normalized LF $\left(i n d_{3}\right)$ and HF $\left(i n d_{5}\right)$ indicators which led to indicators $i n d_{6}$ and $i n d_{7}$. The ratios $L F /(M F+H F)\left(\right.$ ind $\left._{8}\right)$ and $L F / H F\left(\right.$ ind $\left._{9}\right)$, known to be indexes of relative sympathovagal balance, were also considered.

b) Pole based indicators: The originality of this work is to use indicators extracted from the main pole parameters. The indicators are computed from the pole's frequencies, modules and residues of the poles belonging to the upper half of the unitary circle and that are not located at the DC frequency. Let $f_{k}, m_{k}$ and $P_{k}$ be the frequency, module and power, respectively, computed from (5), (6) and (7), for each one of the $M$ poles $z_{k}$ belonging to the upper part of the unitary circle. Here are the pole-based indicators considered in this work:

- The mean, variance and maximal values of the $M$ pole modules $m_{k}$ form indicators $i n d_{10}$, ind $d_{11}$ and $i n d_{12}$, respectively.

- The mean and maximal values of the $M$ pole spectral power $P_{k}$ are indicators $i n d_{13}$ and $i n d_{14}$, respectively.

- The total pole's spectral power calculated as $\sum_{k=1}^{M} P_{k}$ is indicator $i n d_{15}$.

- The mean and minimal value of the $M$ pole's frequencies $f_{k}$ are indicators $i n d_{16}$ and $i n d_{17}$, respectively.

- The frequency belonging to the unitary circle's closest pole is indicator $i n d_{18}$.

- The frequency where is located the pole which has the maximal value of power spectral pole $P_{k}$ form the indicator $i n d_{19}$.

- Finally, the AR modeling error constitutes indicator ind $_{20}$.

\section{RESULTS}

We mentioned in Section II-A that the extracted features' evaluation is based on the umbilical cord artery $\mathrm{pH}$ which is the most commonly used outcome measure, sign of respiratory hypoxia. Umbilical $\mathrm{pH}$ values lower or equal to 7.05 is the widely used value for distinction between pathological and normal delivery [3].

As explained in Section II-C, in this work 20 indicators are used for extracting features from four filtered versions of the 

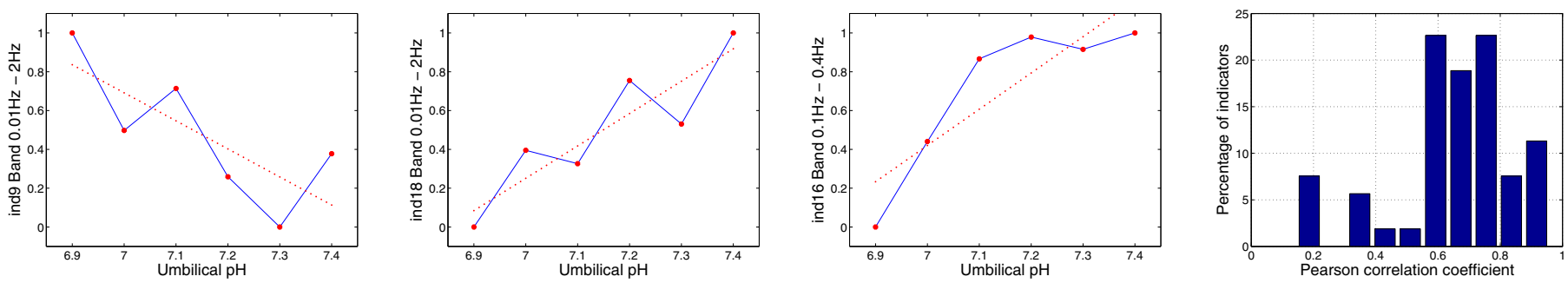

Fig. 1. The mean value (solid line) for 3 different extracted features in function of the $\mathrm{pH}$ with the respective regression model (dotted line). From left to right: AR spectrum-based and AR pole-based indicators features of Band 1, and one R pole-based indicator features of Band 3 . At the far right: the Pearson correlation coefficient for all 53 computed indicators.

FHR signal. The indicators are divided in two groups (AR spectral-based and AR pole-based), where the 20 indicators are used only with the filtered FHR signal in the Band 1 , while only the pole-based indicators are used in the remaining frequency bands. Therefore, 53 features are finally extracted from the FHR signal (20 features from filtered Band 1 signal and 11 features for each one of the other bands). For evaluating the significance of these 53 extracted features, each one of the features is put in correlation with the umbilical $\mathrm{pH}$ values through a scatter plot. This is done by separating the CTG recordings belonging to the CTU-UHB CTG database in groups by $\mathrm{pH}$ value and by calculating the mean of the features for each group. Then, the feature's mean values for each group of $\mathrm{pH}$ value are normalized between 0 and 1 and then are plotted in function of the corresponding $\mathrm{pH}$ which values are set as shown in Table II:

TABLE II

DEFINITION OF THE PH VALUES GROUPS

\begin{tabular}{|c||c|c|c|c|c|c|}
\hline Denoted by $\mathrm{pH}=$ & 6.9 & 7.0 & 7.1 & 7.2 & 7.3 & 7.4 \\
\hline \hline if $\mathrm{pH}<$ & 6.80 & 6.95 & 7.05 & 7.15 & 7.25 & 7.35 \\
\hline and $\mathrm{pH} \leq$ & 6.95 & 7.05 & 7.15 & 7.25 & 7.35 & 7.50 \\
\hline
\end{tabular}

From the 53 FHR signals' extracted features, 29 features show a good correlation with the $\mathrm{pH}$ value after visual inspection of the correlation scatter plots, supported by the computation of the Pearson correlation coefficient on the linear regression of the scatter plot (see the last plot of Figure 1). From the first 3 plots of Figure 1 showing one AR spectrum-based and two AR pole-based features, it is possible to observe a direct or inverse correlation between the feature and the $\mathrm{pH}$ value. In the band $[0.01 \mathrm{~Hz}-2 \mathrm{~Hz}]$, the inverse relationship between ind $_{9}$ and $\mathrm{pH}$ would suggest that when $\mathrm{pH}$ value increases, the HF PSD energy increases with respect to the LF PSD energy, while the direct relationship of the indicator $i n d_{18}$ shows that when the $\mathrm{pH}$ value increases, the frequency of the most stationary pole also increases. In the band $[0.1 \mathrm{~Hz}-0.4 \mathrm{~Hz}]$, the direct relationship between ind $_{16}$ and $\mathrm{pH}$ suggests that the mean frequency of the poles has a growing trend.

\section{Discussion}

The obtained results show that from the 53 extracted features, 29 presented good correlation with the umbilical
$\mathrm{pH}$ and that the extracted AR pole-based features were more significant that the generally used AR spectral-based ones. Finally, it is important to observe that the good correlated features show a big difference between a high $\mathrm{pH}(\mathrm{pH}>$ $7.35)$ and a low one $(p H \leq 6.95)$.

Comparison of the proposed method with current clinical practice in order to improve its potential predictive improvement, physiological interpretation of the features issued from the AR's pole-based analysis and investigation on statistical differences between pathological and normal delivery of the proposed indices for classification algorithms' development are some of the research perspectives provided by this work.

\section{REFERENCES}

[1] G. Rooth, A. Huch, and R. Huch, "FIGO news: guidelines for the use of fetal monitoring," Int J Gynecol Obstet, vol. 25, pp. 159-67, 1987.

[2] G. Georgoulas, D. Stylios, and P. Groumpos, "Predicting the risk of metabolic acidosis for newborns based on fetal heart rate signal classification using support vector machines," Biomedical Engineering, IEEE Transactions on, vol. 53, no. 5, pp. 875-884, 2006.

[3] J. Spilka, G. Georgoulas, P. Karvelis, V. P. Oikonomou, V. Chudáček, C. Stylios, L. Lhotská, and P. Janku, "Automatic evaluation of fhr recordings from ctu-uhb ctg database," in Information Technology in Bio-and Medical Informatics. Springer, 2013, pp. 47-61.

[4] M. Cesarelli, M. Romano, and P. Bifulco, "Comparison of short term variability indexes in cardiotocographic foetal monitoring," Computers in biology and medicine, vol. 39, no. 2, pp. 106-118, 2009.

[5] J. Van Laar, M. Porath, C. Peters, and S. Oei, "Spectral analysis of fetal heart rate variability for fetal surveillance: review of the literature," Acta obstetricia et gynecologica Scandinavica, vol. 87, no. 3, pp. 300306, 2008.

[6] J. Y. Kwon, I. Y. Park, J. C. Shin, J. Song, R. Tafreshi, and J. Lim, "Specific change in spectral power of fetal heart rate variability related to fetal acidemia during labor: comparison between preterm and term fetuses," Early human development, vol. 88, no. 4, pp. 203-207, 2012.

[7] M. G. Signorini, G. Magenes, S. Cerutti, and D. Arduini, "Linear and nonlinear parameters for the analysisof fetal heart rate signal from cardiotocographic recordings," Biomedical Engineering, IEEE Transactions on, vol. 50, no. 3, pp. 365-374, 2003.

[8] M. Romano, P. Bifulco, M. Cesarelli, M. Sansone, and M. Bracale, "Foetal heart rate power spectrum response to uterine contraction," Medical and Biological Engineering and Computing, vol. 44, no. 3, pp. 188-201, 2006.

[9] P. A. Warrick, E. F. Hamilton, D. Precup, and R. E. Kearney, "Classification of normal and hypoxic fetuses from systems modeling of intrapartum cardiotocography," Biomedical Engineering, IEEE Transactions on, vol. 57, no. 4, pp. 771-779, 2010.

[10] V. Chudáček, J. Spilka, M. Burša, P. Janku, L. Hruban, M. Huptych, and L. Lhotská, "Open access intrapartum CTG database," BMC pregnancy and childbirth, vol. 14, no. 1, p. 16, 2014.

[11] L. T. Mainardi, A. M. Bianchi, G. Baselli, and S. Cerutti, "Poletracking algorithms for the extraction of time-variant heart rate variability spectral parameters," Biomedical Engineering, IEEE Transactions on, vol. 42, no. 3, pp. 250-259, 1995. 